\title{
Spectral (Finite) Volume Method for One Dimensional Euler Equations
}

\author{
Z.J. Wang (zjw@egr.msu.edu) \\ Associate Professor of Mechanical Engineering \\ Michigan State University, 2555 EB, \\ East Lansing, MI 48824, U.S.A.
}

\author{
Yen Liu (liu@nas.nasa.gov) \\ Research Scientist \\ NASA Ames Research Center \\ Moffet Field, CA 94035, U.S.A.
}

\section{Basic Idea}

Consider a mesh of unstructured triangular cells. Each cell is called a Spectral Volume (SV), denoted by $S_{i}$, which is further partitioned into subcells named Control Volumes (CVs), indicated by $C_{i, j}$, as shown in Figure la. To represent the solution as a polynomial of degree $m$ in two dimensions (2D) we need $N=(m+1)(m+2) / 2$ pieces of independent information, or degrees of freedom (DOFs). The DOFs in a SV method are the volume-averaged mean variables at the $N$ CVs. For example, to build a quadratic reconstruction in $2 \mathrm{D}$, we need at least $(2+1)(3+1) / 2=6$ DOFs. There are numerous ways of partitioning a SV, and not every partition is admissible in the sense that the partition may not be capable of producing a degree $m$ polynomial. Once $N$ mean solutions in the CVS of a SV are given, a unique polynomial reconstruction can be obtained from

$$
p_{i}(x, y)=\sum_{j=1}^{N} L_{j}(x, y) \bar{u}_{i, j},
$$

where $p_{i}(x, y) \in P_{m}$ (the space of polynomials of degree $m$ or less), $L_{j}(x, y) \in P_{m}, j=1, \cdots, N$ are the "shape" functions satisfying

$$
\int_{C_{i, j}} L_{k}(x, y) d V=\left|C_{i, j}\right| \delta_{j k} .
$$

This high-order polynomial reconstruction facilitates a high-order update for the mean solution of each $\mathrm{CV}$. Consider the following hyperbolic conservation law

$$
u_{t}+\nabla \bullet F=0 \text {, }
$$

where $F$ is called the flux vector. A high-order update can be obtained by integrating (3) in each $C V$ to obtain

$$
\frac{d \bar{u}_{i, j}}{d t}\left|C_{i, j}\right|+\sum_{r=1}^{K} \int_{A_{r}}(F \bullet \boldsymbol{n}) d A=0,
$$

where $\mathrm{K}$ is the total number of faces in $C_{i, j}$, and $\bar{u}_{i, j}$ is the volume-averaged solution at $C_{i, j}$. The flux integral in (4) is then replaced by an $m^{\text {th }}$ order Gauss-quadrature formula

$$
\int_{A_{r}}(F \bullet \boldsymbol{n}) d A \approx \sum_{q=1}^{J} w_{r q} F\left(\boldsymbol{u}\left(x_{r q}, y_{r q}\right)\right) \bullet \boldsymbol{n}_{r} A_{r},
$$

where $J$ is the number of quadrature points on the $r$-th face, $w_{r q}$ are the Gauss quadrature weights, $\left(x_{r q}, y_{r q}\right)$ are the Gauss quadrature points. Since the reconstructed polynomials are piece-wise continuous, the solution is usually discontinuous across the boundaries of a SV. The fluxes at the interior faces can be computed directly based on the reconstructed solution at the quadrature points. The fluxes at the boundary faces of a SV are computed using approximate Riemann solvers given the left and right reconstructed solutions, i.e.,

$$
F\left(u\left(x_{r q}, y_{r q}\right)\right) \bullet \boldsymbol{n} \approx F_{\text {Riem }}\left(p_{i}\left(x_{r q}, y_{r q}\right), p_{i, r}\left(x_{r q}, y_{r q}\right), n_{r}\right),
$$

where $p_{i, r}\left(x_{r q}, y_{r q}\right)$ is the reconstructed polynomial in a neighboring SV sharing face $r$ with the $S V$ in consideration, $S_{i}$. It has been shown [1] that resultant order of accuracy of this SV scheme is $(m+I)$-th order. In addition, the scheme is compact in the sense that a high-order polynomial is reconstructed in each SV without using any data from neighboring SVs. This property can potentially translate into significantly reduced communication cost compared to a high-order k-exact finite volume (FV) method [2] when implemented on parallel computers. In addition, the SV method is much more efficient than the k-exact method because its reconstruction can be solved analytically, and the reconstruction is universal for the same partition, irrespective of spectral volume shapes. The reconstruction problem in a k-exact method must be solved numerically for each cell because the reconstruction stencil is different for each cell, as shown in Figure $\mathrm{Ib}$. 
The SV method is similar to the Discontinuous Galerkin (DG) [3] method in philosophy. However, the SV method has the following advantages compared to the DG method: 1) better stability limit ; 2) unknowns updated independently and no mass matrix inversion; 3 ) only $\mathrm{m}^{\text {th }}$ order surface integration required for $(\mathrm{m}+1)^{\text {th }}$ order schemes, not $2 \mathrm{~m}^{\text {th }}$ order surface and volume integrations as in the DG method; 4) higher-resolution for discontinuities because limiters can be designed for the CV means rather than the SV means; 5 ) the avoidance of a high-order volume integral, which is required in the DG method.

\section{Some Results}

Through simple analysis and numerical tests, it turns out the accuracy, stability and convergence property of the SV method hinges on the proper partition of a SV into CVs. In one-dimensional tests, it has been shown that a uniform partition of a SV is not convergent for higher than fourth-order SV schemes. A partition using the Gauss-Lobatto points has given excellent results. An accuracy study has confirmed the expected high-order accuracy with the SV method.

The SV method has been successfully demonstrate for both 1D and 2D scalar conservation laws [1]. In this paper, the SV method is further extended to the one-dimensional system - the Euler equations. Roe's Riemann solver is employed to compute the flux across SV boundary faces. One demonstration case of blast wave interaction is shown in Figure 2a, which presents the computed density profile with a third-order SV method. The solid line represents a "converged" solution using a MUSCL scheme on a very fine mesh. The computational density profile with a thirdorder DG scheme is shown in Figure $2 \mathrm{~b}$. Note that the SV scheme has far better resolution than the DG scheme.

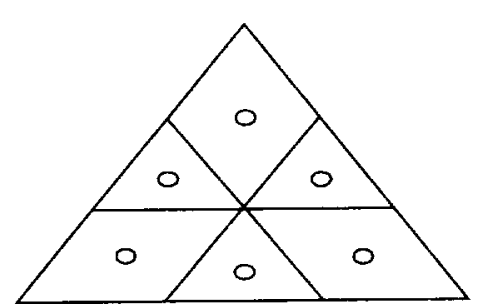

(a)

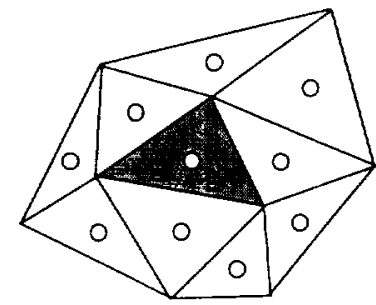

(b)

Figure 1. (a) The partition of a Spectral Volume into six Control Volumes supporting quadratic reconstruction; (b) A possible reconstruction stencil for a quadratic reconstruction in a high-order finite volume scheme;

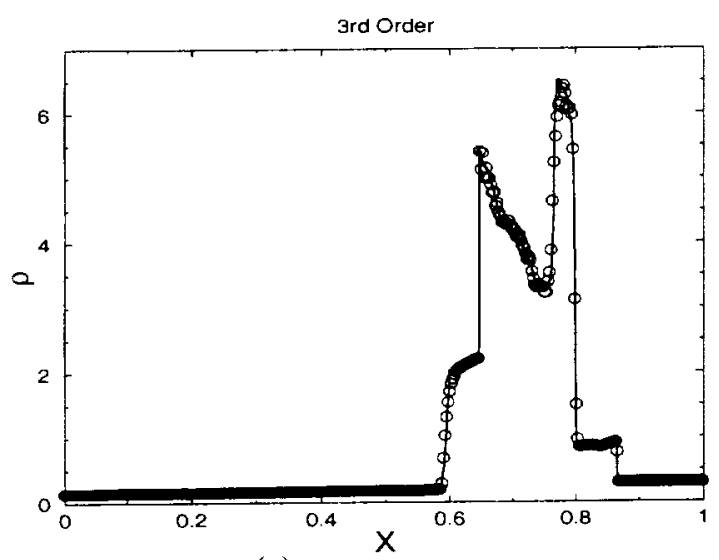

(a)

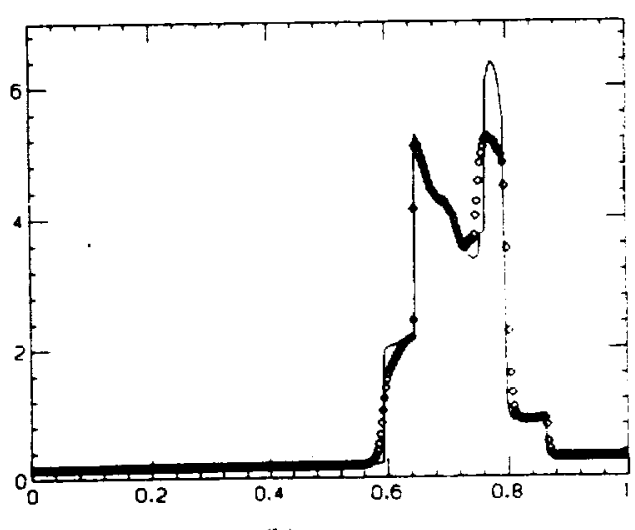

(b)

\section{References}

Figure 2. Third-Order SV Method (a) and DG Method (b) with TVD Limiters, 400 Cells

1. Z.J. Wang and Yen Liu, "Finite spectral volume method for conservation laws on unstructured grids: $2 \mathrm{D}$ scalar equations," submitted to J. Comput. Phys.

2. T.J. Barth and P.O. Frederickson, "High-order solution of the Euler equations on unstructured grids using quadratic reconstruction," AIAA Paper No. 90-0013, 1990.

3. B. Cockburn, S.-Y. Lin and C.-W. Shu, "TVB Runge-Kutta local projection discontinuous Galerkin finite element method for conservation laws III: one-dimensional systems," J. Comput. Phys. 84, 90-113 (1989). 
